\title{
CIGRE/CIRED JWG C4.112 - Power Quality Monitoring
}

M.H.J. Bollen ${ }^{1}$, J.V. Milanović ${ }^{2}$ and N. Čukalevski ${ }^{3}$

\author{
${ }^{1}$ Luleå University of Technology \\ Electric Power Engineering, Skellefteå, Sweden \\ math.bollen@1tu.se \\ ${ }^{2}$ University of Manchester, Manchester, UK \\ ${ }^{3}$ Mihailo Pupin Institute, Serbia
}

\begin{abstract}
In a response to the renewed interest in power quality monitoring and recognising cross-boundary relevance of power quality monitoring, CIGRÉ Study Committee C4 and CIRED established, in late 2010, the Join Working Group (JWG) C4.112: "Guidelines for Power quality monitoring measurement locations, processing and presentation of data". The JWG started work in February 2011 with the aim to address the application aspects of power-quality monitoring, in particular what to measure, how to measure and how to handle recorded data. This paper presents some of the results of the JWG achieved between February 2011 and December 2013, provides recommendations with respect to power quality monitoring depending on identified objectives of monitoring and identifies the areas requiring further development and research in order to comprehensively address the issue of power quality monitoring in contemporary and future power networks.
\end{abstract}

\section{Key words}

Electric power systems, power quality, power quality monitoring, data acquisition and processing.

\section{Introduction}

There has been noticeable increase in the amount of power quality monitoring taking place in electric power systems in recent years. Monitoring of voltages and currents gives the network operator information about the performance of their network, both for the system as a whole and for individual locations and customers. There is also pressure from the customers and the regulatory agencies to provide information on the actual power quality level. Developments in enabling technology (monitoring equipment, communication technology, and data storage and processing) have made it possible to monitor at a large scale and to record virtually any parameter of interest. The change in types of loads connected to the network and proliferation of nonconventional (power- electronicinterface-connected) generators as well as envisaged further increase in non-conventional types of loads/storage (e.g., electric vehicles) puts additional pressure on network operators to monitor and document various aspects of network performance. While many network operators are installing monitoring equipment and while more and more manufacturers have monitors available, there is a lack of knowledge and agreement on a number of aspects of the monitoring process and in particular on processing the recorded data. The users of the data, be it network operators or their customers, are increasingly asking for useful information rather than just large amounts of data to be provided by installed monitors and supporting software.

In a response to this renewed interest in power quality monitoring and recognising cross-boundary relevance of power quality monitoring CIGRE / CIRED Joint Working Group C4.112 (hereafter abbreviated as JWG) was established and started its activities in 2011 with a three-year mandate to deliver a CIGRE technical brochure containing guidelines for monitoring power quality in contemporary and future power networks. The technical brochure addresses the three basic questions for any power quality monitoring system/campaign, namely, what to measure, how to measure and how to process and report recorded data. At the time of writing of this paper (February 2014) the writing of the brochure is in its final stages [1].

This paper summarises some of the results of the JWG achieved between February 2011 and February 2014. Depending on the main purpose and objectives of the monitoring, different strategies are outlined in the report and straightforward methods and examples are provided for end-users. Monitoring goals range from compliance verification and troubleshooting up to advanced applications and studies. Other important factors that must be considered by an engineer designing a power quality (PQ) monitoring system include the total number of monitoring sites, their location, available resources and the approach to data storage and processing. For each of the identified monitoring objectives the most important issues are described: type of monitoring (continuous, short-term), monitoring location, monitored parameters, sampling rate, averaging window, telecommunication and data handling infrastructure. Finally, the paper gives the main recommendations of the JWG and discusses some of the key results. 


\section{Motivation for $P Q$ monitoring}

Virtually all aspects of a PQ monitoring deployment are influenced by the objective(s) that the utility is seeking to address. As such, the single most important step in deployment of a PQ monitoring system is clear identification of that system's objective(s). In general, the following 6 main objectives for PQ monitoring can be distinguished (not necessarily given in order of importance):

1. To verify compliance with standards - Compliance verification compares a defined set of PQ parameters with limits given by standards, rules or regulatory specifications. In most cases a minimum of two stakeholders is involved and at least some results are reported externally. For utilities, the economic drivers may include regulatory penalties and incentives associated with PQ compliance and improvements, along with the costs associated with disputes.

2. To assess the performance of the system - Performance analysis is usually an issue for a network operator and results are used primarily for various internal purposes (e.g. strategic planning, asset management, etc.)

3. To characterise a particular site - Site characterisation is used to quantify and describe PQ at a specific site in a detailed way.

4. Troubleshooting - Troubleshooting measurements are always based on a PQ problem (e.g. exceeding levels, equipment damage); usually there is a specific initiating event for a troubleshooting measurement. This may follow a compliance verification measurement, if limits are not met. Customer complaints arise from trips or other disruption to their processes. For customers, poor PQ performance leading to interruption of production can be expensive, particularly if critical process loads are being adversely affected.

5. Advanced applications and studies - Advanced applications and studies are growing in popularity due to the higher resolution and complexity of the data and its more timely communication. Advanced studies include more specific measurements and analyses that are often not part of the daily business.

6. Active power quality management - Active PQManagement includes all applications where any kind of network operation control is derived from the PQ measurement results. This may be offline or real-time control.

\section{Existing practice}

Market and business forces, including initiatives related to smart grids and performance based rating, have increased the need for network operators to understand the true performance of their transmission and distribution networks. Almost all utilities monitor the PQ on their system to some extent. The system-wide monitoring of PQ in each utility is heavily influenced by its regulatory environment. Regarding temporal aspects of the deployment of PQ monitoring, the following approaches are part of existing practice:
Long-term, continuous PQ monitoring, with fixed instrument installation

PQ monitoring with portable instruments, with a rotating approach, where a monitor stays at a site for a specific period of time to capture a sample of measurements and then is moved to another site

Temporary and short-term PQ monitoring, with mobile or handheld instruments, mostly for a period of time sufficient for problem identification (i.e., for troubleshooting).

A questionnaire [2] on power quality monitoring practices has been developed by JWG and distributed to a large number of transmission (TSO) and distribution system operators (DSO) from 43 countries on all continents. (The general term "utility" will be used in this paper.) A total of 114 responses were obtained. Some of the results from the survey are summarized below; further details are found in [2].

\section{A) Location selection}

PQ monitoring locations can be chosen according to: i) the voltage level; ii) one of the power system physical elements e.g., substation, feeder (typically MV), end user connection point (i.e., Point of Common Coupling); iii) the availability of monitoring equipment and/or suitable transducers.

From the 114 responses to the questionnaire [1] it was found that a typical utility has fixed PQ monitors at a number of sites $(55 \%$ of TSOs and $40 \%$ of DSOs have monitors at more than $10 \%$ of their sites) and also carries out monitoring with portable units both to investigate end user complaints and to assess the system as a whole. See Figure 1 for details.

Several criteria are used within the power industry at present, to select specific locations for installing permanent PQ monitors, including:

$\checkmark$ Random selection of the monitoring sites

$\checkmark$ Monitoring at required sites as defined by the regulator

$\checkmark$ Monitoring at a number of sites such that a statistically representative sample is achieved

$\checkmark$ Selection based on identified or reported power quality complaints

$\checkmark$ Monitoring at sites where important and/or sensitive customers are, or will be connected

$\checkmark$ Monitoring at sites with expected high levels of PQ disturbances

$\checkmark$ Monitoring at sites that are significant for the operation of the system 

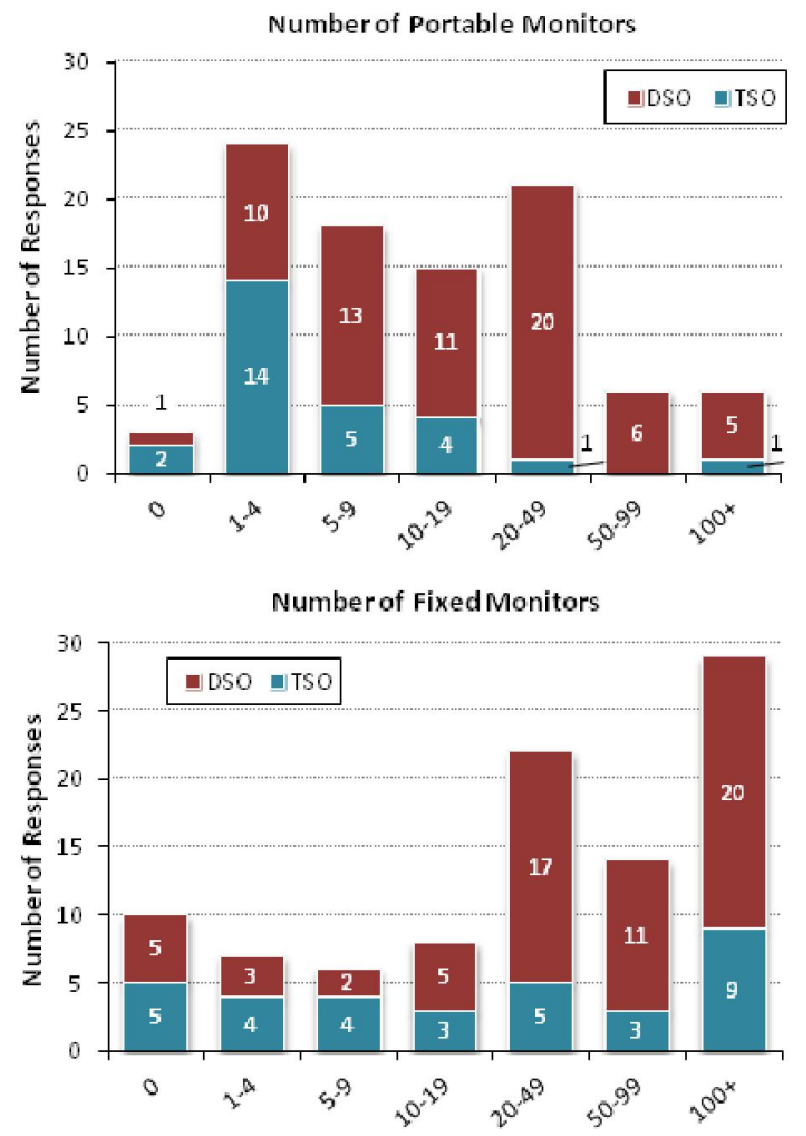

Figure 1: Number of portable and fixed monitors for DSOs and TSOs.

\section{B) Parameters monitored}

The types of disturbances and parameters to be monitored depend on the objectives and the way in which the information will be used. Any PQ monitoring system specification requires three choices

$\checkmark$ the parameter to be calculated (for example the steady-state voltage, or the THD)

$\checkmark$ the objective values (for example the 95-percentile, across a week, of 10-minute averages)

$\checkmark$ the limit value

An example of the related results from the survey is shown in Figure 2.

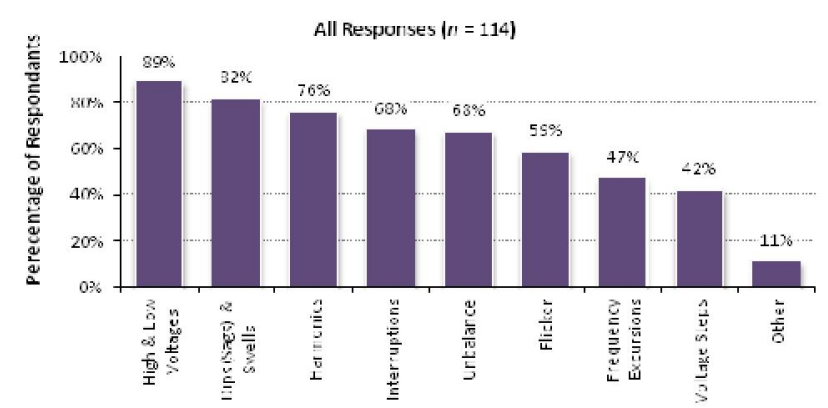

Figure 2: Types of disturbances monitored

In the case of monitoring to assess compliance, the parameters to be monitored depend on the standard or regulation which is to be applied. Most standards only call for compliance with respect to voltage disturbances, e.g.,
EN 50160 [4] and IEC 61000-3-6. The most common parameters monitored are frequency, RMS voltage, voltage unbalance, voltage harmonics and voltage dips and swells. Some national standards such as IEEE 519 require measurement of current parameters as well as voltages.

\section{Presentation and Reporting of Monitoring Results}

Monitoring to assess the compliance involves comparing statistical indicators against relevant limits. In this case, it may be sufficient for the assessment to be made automatically and a report merely to confirm compliance. An alternative method, that is also widely used, is the "report by exception." In this case, a report is only produced when non-compliance is detected by the monitor. Describing the performance of the system involves displaying or summarising the monitored data from many sites. The main concern is to provide an appropriate summary.

When considering an individual site, the most common practice is to monitor and report the same indices for site characterization as for compliance monitoring. Another widely used method of displaying a continuously-varying parameter at a site is a histogram plot showing the proportion of time for which the parameter is at particular value. The IEC 61000-4-30 standard [5] gives recommendations on which indices to use for contractual applications.

Although benchmarking is inherently difficult between systems of widely different size and structure, this would be helped by the adoption of standardised approaches or some widely used methods. Harmonisation of monitored parameters is essential to allow benchmarking. Several steps towards such harmonisation have been made in the past: the IEEE standard on power quality monitoring, IEEE 1159 [3]; the European standard for voltage-quality characteristics, EN 50160 [4]; and the IEC standard on power-quality monitoring, IEC 61000-4-30 [5]. More recently a set of parameters and indices for benchmarking was proposed in [6].

\section{Selection of monitoring locations}

Power quality monitoring locations are strongly related to the power system architecture and infrastructure and also to monitoring purposes. In classic power systems PQ monitoring points are usually located at the interfaces between the four fundamental component segments: conventional generation, transmission, distribution and customer. In future power networks with large penetration of RES/DG a new layer of monitoring might be added at interconnection points of these new types of energy sources. Lately, technologies including measurement transformers, Intelligent Electronic Devices (IED) and communications have undergone important evolution which has already impacted to a certain extent the PQ monitoring and the selection of monitoring locations. In future grids, the place of the conventional PQ analysers will be gradually taken by constantly improving IED's, featuring some power quality 
functionalities, for example: relays, controllers, Remote Terminal Units (RTU), Phasor Measurement Units (PMU), digital multifunctional meters, etc. augmented by Global Positioning System (GPS) devices.

\section{A) Recommendations}

For extra high-voltage (EHV) and high-voltage (HV) networks, the existing practice, measuring at all $\mathrm{EHV} / \mathrm{HV}$, EHV/MV and HV/MV substations and at the connection points of all EHV and HV customers, producers (power stations) and consumers (industrial customers) will continue to be used. Monitoring in the EHV and HV networks should be long-term and continuous at all measuring locations and performed by fixed, permanent PQ monitors.

In MV networks, the PQ should be permanently monitored on the MV side of the transformer in all EHV/MV and HV/MV substations. For MV customers, the measurement location should be at the point of connection to the grid (PoC) or at a convenient location close to the PoC.

In LV networks, PQM should be performed at the PoC of a selection of sensitive customers, as this will give a statistically relevant picture of PQ for all customers if a sufficient number of locations are used.

\section{B) Metering Infrastructure}

In the future networks, the Advanced Metering Infrastructure (AMI) will play a bigger role in PQ monitoring than it plays today. Intelligent, most likely three phase meters located at the $\mathrm{PoC}$ of industrial and commercial customers and equipped with PQ acquisition and processing capabilities and complying with national and international standards will be integrated in distribution PQ monitoring systems.

Currently available single-phase household smart meters, even if equipped with PQ functionalities, are able to detect only a limited set of voltage disturbances, namely the supply voltage variations. From the point of view of PQ monitoring objectives/goals, the following guidelines were proposed.

\section{C) Compliance verification}

The PQ compliance is generally carried out at the boundaries determined by the chain of power delivery which implies different voltage levels (EHV, HV, MV, LV). For compliance assessment, the monitoring location selection should be governed by the following general considerations:

i) PQ monitoring locations are distributed between voltage levels according to number of customers, reported PQ issues, customer sensitivity to different disturbances, etc.

ii) ii) The number of intermediary substations between any customer and any monitoring location should not exceed two substations (two voltage transformations).
The precise location of the instrument in each selected substation should be decided based on the availability of appropriate transducers allowing accurate 3-phase measurements (particularly important in the case of harmonic measurement) and in case that appropriate transducers are not available or not possible to install at desired location, monitoring should be performed at nearby substations equipped with required transducers or substation where installation of such transducers is feasible.

\section{D) Performance assessment}

In general, for performance comparison, there are two different approaches which should be used for selection of monitoring locations: i) Selection of entire population (all sites). This is recommended for $\mathrm{HV}$ or $\mathrm{EHV}$ transmission grids with a reasonable number of substations or number of connected customers; ii) Selection of a representative number of sites either using statistical methods or methods based on analysis of network characteristics.

\section{E) Site characterisation}

The objective of PQ monitoring for site characterisation is either to predefine the expected power quality to a potential customer or to assess and verify PQ once the customer is already connected to the grid. In case the customer is not yet connected, PQ can only be evaluated without the impact of the new customer. The closer PQ is measured to the future connection point, the better the approximation for potential customers. The most convenient method for verification of performance by existing customer is to measure PQ parameters in parallel with power revenue meters. In this case there is no need to install new measurement transformers.

\section{F) Troubleshooting}

The objective of troubleshooting is to identify why one or more devices installed at customer's site do not operate as intended. The best option for troubleshooting monitoring would be to measure voltage and current as close as possible to the concerned equipment. The recommended practice for troubleshooting uses measurement data from 3 different locations

i) At the terminal of the equipment that failed or at a close by terminal

ii) At the PoC of the equipment's owner

iii) At the PoC of a close by customer or the busbar in the substation supplying the customer with the faulty equipment (historic measurements from network operator can be used for this purpose as well).

In some cases network operators are performing troubleshooting within their operational area.

\section{G) Advanced applications and studies}

Regarding advanced applications and studies, some of the smart transmission and distribution applications and infrastructure components can in addition to their main use provide PQ related information according to their 
capability to monitor PQ. So, the monitoring location is dependent on the location of these advanced data acquisition devices present in substations and along the feeders. Although data acquisition infrastructure and devices are a convenient source of PQ related information, there present limitations in terms of PQ data acquisition, in comparison with dedicated PQ devices, must be kept in mind. Finally, for active PQ management, in most cases the PQ should be monitored at the PoC of low voltage or medium voltage customers.

\section{Monitoring parameters}

Once the monitor locations are decided one has to select which PQ disturbances are going to be recorded, which parameters to monitor, how to store and transmit recorded data and what should be the accuracy of transducers. This section provides more details with regards to these issues.

\section{A) Number of monitors}

There is a number of PQ monitoring issues that depend not only on the objective of PQ monitoring but also on the number of monitors involved.

When up to a dozen monitors are involved there is typically no need to reduce the number of parameters to be monitored. In such cases sophisticated monitors equipped with state-of-the-art proprietary software for data processing are typically deployed with no need for specific communication links or large IT systems.

The current trend however, seems to be to deploy hundreds or even thousands of monitors. With such monitoring programs the IEC 61000-4-30 Class A monitors should be used. The trade-off between costs and functionally becomes important in this case. The use of Class A monitors will ensure comparison of results from different monitoring programs and exchange of knowledge and experience between programs. Communication and data storage are also very important in this case and flat and open formats for data handling and efficient and open communication protocols for data transfer should be used.

Considering massive roll-out of smart meters in several countries, it is very likely that monitoring programmes in the future could involve millions of monitoring units having diverse recording and data processing capabilities. However, not all smart meters with PQ functionality, currently on the market, measure PQ in an appropriate way. Cheap devices should be developed that measure supply voltage variations, interruptions, voltage dips and voltage swells, according to IEC 61000-4-30 Class A. Communication protocol, data storage and handling become essential in such programs as well as the data to be recorded and transferred to the central or distributed database.

\section{B) Parameters to be recorded}

For compliance assessment the parameters to be recorded are determined by the standard or regulation that is to be applied, for example EN 50160. The most common parameters are rms voltage, voltage unbalance, voltage harmonics, voltage dips and voltage swells. When compliance of a customer with connection agreements is verified, current-related parameters should be measured.

For benchmarking and performance assessment, the choice of parameters is similar to the one for compliance assessment, sometimes even more limited as not all parameters may be of interest to the utility. A recommendation on parameters for benchmarking of sites and systems is given in [6].

For site characterisation a wider range of parameters should be monitored. In addition to parameter averages over certain periods, maximum and minimum values could be also monitored. Sometimes it is also appropriate the measure over shorter time windows than 10 minutes. Supply voltage variations (preferably $1 \mathrm{~min}$ average or less) should be monitored for all MV and LV locations. Voltage swells should be measured for all locations. Voltage dips should be measured for all locations with industrial or commercial customers. Harmonics and flicker should be measured when there is a specific interest, either because of sensitivity of equipment or because of expected high levels.

For troubleshooting a wide range of parameters should be measured, beyond those that are standardized. The specific selection depends strongly on the type of problem that has to be solved.

\section{C) Data resolution}

For compliance assessment, benchmarking and performance analysis the 10-minute averages as defined in IEC 61000-4-30 are sufficient for steady-state disturbances. For voltage dips and swells, magnitude and duration should be recorded in all three phases where possible.

For site characterization, shorter time resolution is recommended as this will give important background information in case any limits are exceeded or when levels are close to the limits.

For troubleshooting a high time resolution should typically be chosen.

\section{Presentation of results}

The presentation of the results depends on the objective of the monitoring, but also on the parameters recorded and on the number of monitors involved in the program.

\section{A) Compliance assessment}

The data analysis and reporting intervals are typically set by the regulatory requirements. Where the requirements do not prescribe this, one-week interval is recommended for the data analysis and reporting should be over oneyear period. Next to the basic information (compliance or not), it should be documented which parameters have exceeded the limits, for which periods, and at which 
locations. Information on parameters close to the limit should be also provided.

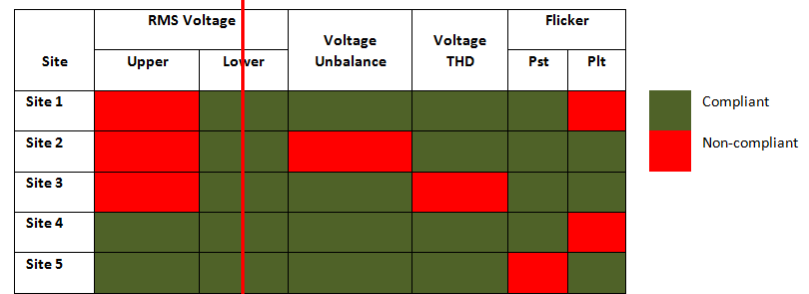

Figure 3: example of reporting of compliance assessment for multiple sites

\section{B) Benchmarking and performance analysis}

Both data analysis interval and reporting interval should be one year. There is no need for obtaining weekly values as in the case of compliance assessment. In some cases, statistical indices may be calculated for every week (in particular for trending and to quantify seasonal variations) followed by a stat stical analysis of the resulting 52 weekly values. Reporting is an important part of benchmarking and performance analysis.
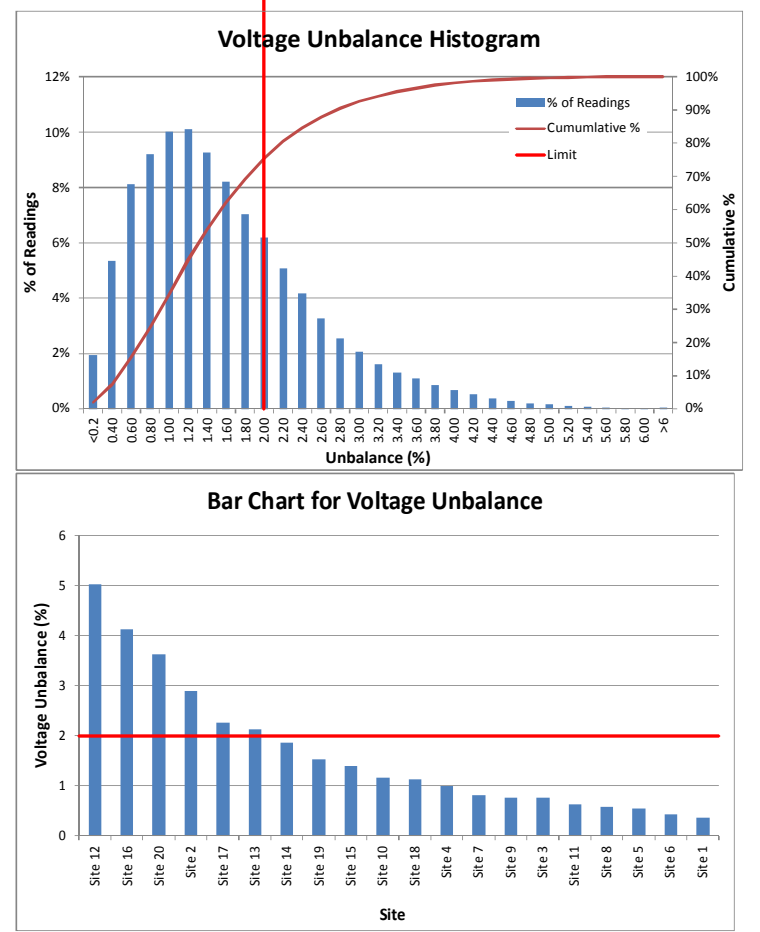

Figure 4. Histogram for measurements at one location (left) and at multiple locations (right)

Graphical representation of the results is often the best for providing quick information. However, the data may be additionally given in tabular format to allow numerical comparisons. Two examples are given in Figure 4. The left-hand graph shows the distribution of the 10-minute unbalance values at one location; the vertical red line indicates the compliance limit. The right-hand graph gives the distribution of the site indices (e.g. $95 \%$ of the 10 minute unbalance over one week) over all monitored sites. The compliance limit is indicated by a horizontal red line. When a large number of sites are involved, a further data reduction may be required. Instead of, or in addition to, distributions over the sites, the sites may be grouped into different types. This may be grouping per utility, but also for example rural versus urban sites or a comparison between sites with domestic, commercial and industrial customers. Trend analysis can be included as well to show seasonal and yearly variations.

\section{C) Site characterisation}

The data analysis and reporting intervals should at least incorporate one cycle of normal activity. This may be normal activity for the customers connected to the site, but also normal activity resulting in the voltage disturbances at the site. Intervals may vary from a few hours (for the emission from industrial installations) up to several years (when yearly variations are expected to be big, as in the case of voltage dips). The reporting methods for this application are partly similar to the ones for benchmarking and performance analysis of individual sites, as shown in the left-hand graph of Figure 4. For voltage dips, scatter plots and voltage-dip tables are suitable ways of presenting the characteristics of a site. Contour charts (Figure 5) may be appropriate for longer monitoring periods, e.g., several years. In addition to statistical distributions, the variation of parameters with time should be also reported.

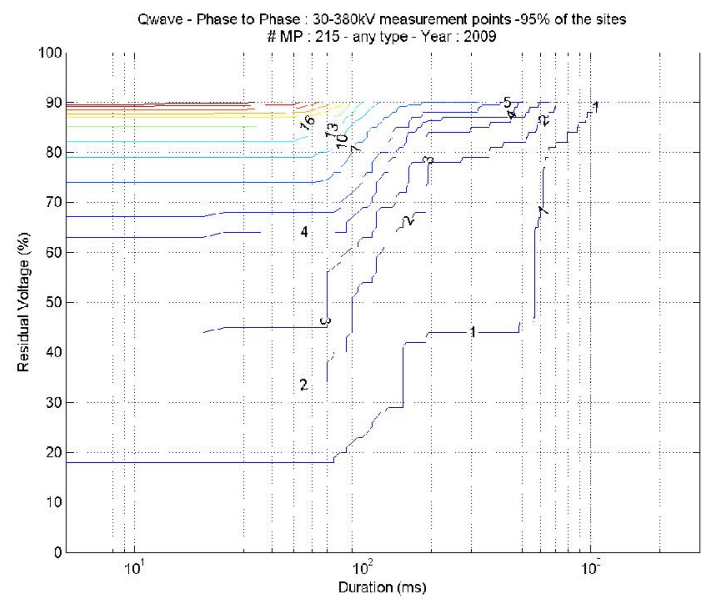

Figure 5. Voltage-dip contour chart for site characterisation; each contour connects points with an equal number of dips per year more severe than the given residual voltage and duration

\section{D) Troubleshooting}

The data analysis and reporting methods and intervals depend strongly on the type of the problem that has to be solved. It may be as short as a few hours (when steadystate disturbances are obviously outside of specified range) or as long as several years (for rare events with severe consequences). It is recommended to perform data analysis on a weekly basis and to allow for up to several weeks of monitoring. When power-quality induced failures have large economic consequences, it is recommended to install permanent monitoring equipment and report on a weekly basis. Statistical distributions and variation of parameters with time, over each oneweek monitoring period, should be included in all reports. 


\section{E) Active Power Quality Management}

Having in mind the basic role of PQ management to initiate network control based on PQ measurement results, it is clear that the PQ measurement (typically harmonics, asymmetry, flicker, etc.) must be integrated in the realtime control loop. These measurements might come from PQM devices integrated with the other components of the PQ control system or they might come from separate, possibly already existing, measuring devices at the same location.

The PQ measurements acquired from this location might be used for site contract verification or site characterisation. Today the most used PQ Management applications are those related to active harmonics filtering and dynamic reactive power compensation. Regarding the data analysis and reporting interval in this case two separate issues exist. One is in the real-time timeframe and thus embedded in the PQ management device itself, with alarm transmission (SCADA) to control-centre operators. Another issue is reporting for the purpose of after the fact analysis. Utility requirements in the later case can be based on presentation and reporting guidelines already defined in this section for different objectives (site characterization and compliance verification probably come first). Regarding reporting methods in case of after the fact analysis, reporting methods applicable for compliance verification or site characterisation might be also used.

\section{Transients - Measurement, Characterisa- tion and Reporting}

\section{A) Definitions}

According to the Merriam-Webster online dictionary transient refers to something "passing especially quickly into and out of existence". The much bigger paper version of Webster's third international dictionary gives as one of the definitions: "a temporary or rapidly changing state or condition of an electrical system; a temporary electrical oscillation that occurs in a circuit because of a sudden change of voltage or of load'. According to the 20000 page Compact Oxford English Dictionary the term was first used in this meaning by Steinmetz in 1911.

The IEC dictionary (www.electropedia.org) defines a transient as "a phenomenon or a quantity which varies between two consecutive steady states during a time interval short compared with the time-scale of interest".

According to the glossary with IEEE Std. 1159 and IEEE Std. 1250, a transient is " a phenomenon or a quantity which varies between two consecutive steady states during a time interval that is short compared to the time scale of interest. A transient can be a unidirectional impulse of either polarity or a damped oscillatory wave with the first peak occurring in either polarity."

EN 50160 does not use the term transient but the term "transient overvoltage", which is a "short duration oscillatory or non oscillatory overvoltage usually highly damped and with a duration of a few milliseconds or less". Note that this is the only definition where a specific time scale is mentioned.

There is however no definition of a transient from a power-quality perspective in the same way as there is for voltage dips, swells and interruptions. Such a definition would require the choice of a voltage or current parameter and their comparison with a threshold.

In this section, we will consider as transients, phenomena up to a few $\mathrm{ms}$ in duration.

\section{B) Measurement}

Certain types of transients contain very high frequencies and these frequencies are an important contribution to the severity of the transient. Examples are transient overvoltages due to lightning; prestrikes and restrikes especially with vacuum switchgear; and transients in gasinsulated substations. For the correct measurement of such transients, special transducers are needed, like resistive voltage dividers. At transmission level, such special transducers are already needed for the accurate measurement of relatively low-frequency transients (500 $\mathrm{Hz}$ and higher). The same limitations hold for the measurement of transients as for the measurement of harmonics. With new installations it is recommended to consider the use or broadband transducers at an early stage. Such transducers are not only needed for measurements of harmonics or transients but also for diagnostic purposes like the detection of partial discharges and for travelling-wave-based methods of fault location.

The voltage to be used for the evaluation of voltage transients or transient overvoltages depends on the application.

$\checkmark$ For insulation coordination, it is recommended to use phase-to-ground voltages as input;

$\checkmark$ For equipment performance, including equipment damage, it is recommended to use the voltage that is most representative for the voltage experienced by the equipment. This will be the phase-to-neutral voltages in directly-earthed low-voltage networks and in directly-earthed medium-voltage networks with single-phase MV/LV transformers. In all other cases, it is recommended to use phase-to-phase voltages.

$\checkmark$ For trouble-shooting in installations with sensitive equipment it is recommended to measures phase-tophase, phase-to-neutral and neutral-to-ground voltages.

\section{C) Low voltage measurements}

In low-voltage networks, the local equipment present can strongly impact the measured voltages and current during transient overvoltages. It is suggested in an informative annex with IEC 61000-4-30 that currents give a better indication of the severity of the transient than the voltages. This is only true in a qualitative sense: a high transient overcurrent might indicate that a downstream 
event takes a large amount of energy. For trouble-shooting purposes this might be important information.

But the current as well as the voltage will be strongly dependent on the equipment present. This makes it difficult to compare different sites and to use the information obtained from a survey to predict future levels, when different equipment may be present. The use of measurements of transient overvoltages and overcurrents in low-voltage networks is therefore of limited use for benchmarking and for site characterization.

Measurements of voltage transients can still be used for benchmarking and site characterization even in lowvoltage network as long as the maximum voltages are below the ignition voltages of the overvoltage protection of the devices present.

\section{D) Event triggering}

Different equipment uses different types of triggering; an overview is given in IEC 61000-4-30 in which six different methods are mentioned (the term "detection method" is used in that standard), where the last is simply described as "other methods". There is no standardized method for the detection of transients, neither voltage transients, nor current transients, nor transient overvoltages or transient overcurrents.

Some instruments allow the user to select a triggering method. In that case the method can be adapted to the kind of phenomenon that is being studied. For example, if the interest is in events with high rate-of-change-of-voltage $(\mathrm{dv} / \mathrm{dt})$, the $\mathrm{dv} / \mathrm{dt}$ should be used as a trigger.

Some instruments use a high-pass filter and trigger when the level of the high-pass signal exceeds a certain value. For trouble-shooting purposes, the actual triggering method is often of less importance, but it is recommended to record waveform for any detected transient.

When the results are used for statistical purposes, like site characterization or trouble shooting, knowledge on the triggering method is important. The selected triggering method can provide a large statistical bias on the results.

\section{E) Event characteristics}

There are many different types of events and it is difficult to characterize all of them by a limited number of characteristics. Also for the event characteristics a list is provided in IEC 61000-4-30. But no further description is given nor any method for calculating those transients. Also here is the list most likely incomplete as most monitor manufacturers use their own methods, for lack of a standard method.

Some instruments do provide waveforms for voltage and currents, whereas others only give the event characteristics. When waveforms are available, it is possible to use different instruments and apply the same calculation method for event characteristics to all events. When no waveforms are available, care should be taken that different instruments use comparable methods. Otherwise it will not be possible to benchmark different locations or to compare otherwise between instruments.

The event characteristics fall into the following groups (description for voltage, but equally valid for current):

$\checkmark$ Magnitude of the event: this can for example be the maximum instantaneous voltage; the maximum deviation from the steady-state waveform; the amount with which the maximum instantaneous voltage exceeds the nominal voltage maximum; or the peak value of a damped sinusoidal to represent the transient.

$\checkmark$ Duration of the event: this can for example be the time above a threshold for the actual voltage; the time above a threshold for the deviation from the steady-state waveform; the time it takes for the voltage to drop to $50 \%$ of its maximum value, or the damping time constant of a damped sinusoid to represent the transient.

$\checkmark$ V-t integral: the integral of voltage or voltage deviation over the duration of the event.

$\checkmark$ Energy of the event: the integral of voltage square or voltage deviation square over the duration of the event.

$\checkmark \quad$ The rate-of-rise of voltage: this can for example be the highest $\mathrm{dv} / \mathrm{dt}$ or the average $\mathrm{dv} / \mathrm{dt}$ during the initial rise of the voltage; it can also be the highest $\mathrm{dv} / \mathrm{dt}$ during the whole event.

Work should be initiated in international standard-setting groups to come with standard methods to calculate these characteristics. Such standards will have to include at least the difference between impulsive and oscillatory transients and the processing of transients in three-phase systems.

\section{F) Site and system performance}

For site characterization and benchmarking it is necessary to agree on methods for characterizing site and system performance. The following recommendations are made for this:

$\checkmark$ As input to the performance, the three indices given in the previous section should be used.

$\checkmark$ Magnitude and duration should be presented in the same way as for voltage dips in a two-dimensional way. For site characterization this might be in the form of a table or in the form of a contour chart. For benchmarking a limited set of site indices should be selected, for example the number of events more severe than a certain curve in the magnitude-duration plane.

$\checkmark \quad$ Site characterization with respect to $\mathrm{dv} / \mathrm{dt}$ should be in the form of a bar chart, a probability distribution function or a similar method. For benchmarking, the number of events exceeding a certain $\mathrm{dv} / \mathrm{dt}$ values should be used.

$\checkmark$ System performance with respect to magnitude and duration should by presented through average and high-percentile values (for example 90 percentile) of the tables or contour charts used to characterize individual sites. 
$\checkmark \quad$ For benchmarking of complete systems, average and high-percentile values of the site indices should be used.

Where multiple sites are involved, e.g. for benchmarking, it is important to ensure that the different instruments used calculate the event characteristics in a comparable way. When waveform data is available, it is recommended that the event characteristics are calculated for all data by the same algorithm. Unless dedicated transducers are used, it is also important that the voltage and current transformers or transducers at the different locations have similar characteristics where it concerns transients.

\section{Future work}

Several important aspects of PQ monitoring were identified by the JWG while preparing the report, but could not be addressed adequately due to limited time and resources. Future work is therefore strongly recommended in the following areas

i) Analysis and reporting methods for discrete disturbances - sags, swells and transients

ii) Identifying appropriate limits for discrete disturbance

iii) Development of standardised methods to calculate characteristics of voltage and current transients. Such methods will have to include at least the difference between impulsive and oscillatory transients and the processing of transients in three-phase systems

iv) Development of simple, robust and standardized methods for the storage and exchange of power quality data, thus allowing interchange of devices from different vendors

v) Reporting techniques for active PQ management and advanced applications and studies. This is particularly required for active PQ management as it is likely to become more required and implement in the future

vi) Computationally efficient techniques for data processing and extraction of relevant PQ information

vii) Data presentation and visualisation software to facilitate on-line tracing and graphical representation of network PQ status. This will facilitate active PQ management and identification of weak areas in the network with respect to $\mathrm{PQ}$.

\section{Acknowledgement}

CIGRE/CIRED Joint Working Group C4.112 consisted of the following members: Jovica V. Milanović, Convenor (GB), Jako Kilter, Secretary (EE), Shaghayegh Bahramirad, Web Officer (US); Richard Ball (GB), Victor Barrera (ES), Math H.J. Bollen (SE), Delmo Correia (BR), Ninel Čukalevski (RS), Anne Dabin (BE), Paul Doyle (IE), Sean Elphick (AU), Paulillo Gilson (BR), Bill Howe (US), Johan Höglund (SE), Jan Meyer (DE), Robert Neumann (IT), Bernard Parent (CA), Jørn SchaugPettersen (NO), Paulo Ribeiro (NL), José Maria Romero
(ES), Nicolas Trinchant (FR), Francisc Zavoda (CA), Liliana Tenti (IT) and the following corresponding members: Emmanuel de Jaeger (BE), Morten Møller Jensen (DK), Kah-Leong Koo (GB), Nuno Melo (PT), Fabio Andrés Pavas Martinez (CO). Further contributors to the final report were: Robin Preece (GB), Jose Manuel Avendano-Mora (US).

\section{References}

[1] Guidelines for power quality monitoring measurement locations, processing and presentation of data, Draft of Final report of CIGRE/CIRED JWG C4.112, December 2013.

[2] J.V.Milanović, J.Meyer, R.F.Ball, W.Howe, R.Preece, M.H.J.Bollen, S.Elphick and N. Cukalevski "International Industry Practice on Power Quality Monitoring", accepted for publication in the IEEE Transactions on Power Delivery, TPWRD-00531-2013 $(13 / 09 / 13)$

[3] IEEE 1159, Recommended Practice for Monitoring Electric Power Quality.

[4] EN 50160, Voltage characteristics of electricity supplied by public electricity networks.

[5] IEC 61000-4-30, Electromagnetic compatibility (EMC) - Part 4-30: Testing and measurement techniques - Power quality measurement methods

[6] Guidelines of good practice on voltage-quality monitoring for regulatory purposes, Council of European Energy Regulators, December 2012. 